\title{
Competitividad de la quinua perlada para exportación: el caso de Puno
}

\author{
Jorge Hilario Guzmán Bautista \\ Universidad Nacional Mayor de San Marcos. Lima, Perú \\ Correo electrónico: iguzmanb@unmsm.edu.pe \\ Recibido: 10/5/2013 / Aprobado: 25/6/2013
}

\begin{abstract}
RESUMEN: La investigación tiene como objetivo principal determinar el volumen de la oferta exportable de la quinua (Chenopodium quinoa willd) perlada, y los factores que hacen de ella un producto competitivo. Para ello, se ha utilizado el análisis de la cadena de valor, el análisis del diamante competitivo de Porter y el análisis de las fortalezas, oportunidades, debilidades y amenazas del sector. Se pudo vislumbrar el nivel en que se encuentra la cadena de la quinua y la brecha que lo separa de lo óptimo, para lograr su desarrollo y atender el incremento de su demanda internacional. Como alternativa de solución se proponen un conjunto de estrategias certeras en el mediano y largo plazo, proyectando una cadena moderna eficaz y competitiva del sector.
\end{abstract}

Palabras clave: competitividad / quinua perlada / oferta exportable

\section{Competitiveness pear quinoa for export: The Puno case}

ABSTRACT: The research study has as main goal to determine the export offer volume of the grain "quinuaperlada", and the factors that make it a competitive product. For this purpose we have used the Chain of Value analysis, the Porter Competitive Diamond analysis and the Strength Weaknesses Opportunities and Threatens analysis. The diagnosis shows the present state and situation of the quinoa in the market as well as the gap still existing between it and the ideal level pursued as a contribution to make this product the base of new business opportunities, able to satisfy the strong increasingly demand for it. According to the suggested solution to the above referred problems, a number of accurate strategic middle and long term decisions have been tested, which mean a proposal for a more efficient and competitive chain in this economic sector.

Keywords: competitiveness / pear quinoa / profer exporting 


\section{INTRODUCCIÓN}

La baja productividad actual de la quinua perlada, su bajo volumen de exportación en los últimos años, así como el uso excesivo de eslabones en la cadena de comercialización hacen que existan dificultades de carácter coyuntural y estructural en la competitividad de la quinua. Se requiere un estudio donde se integren realidades físicas, económicas, tecnológicas y socioculturales de la región para dar alternativas de solución a este problema. Se planteó la necesidad de desarrollar actividades de exportación que generen utilización de mano de obra y que, a la vez, permitan obtener divisas para nuestro país, favoreciendo de manera positiva la balanza comercial. Se justifica la necesidad de crear condiciones productivas capaces de vislumbrar rentabilidad y beneficio en el mediano y largo plazo, de tal forma que se favorezcan otras actividades articuladas con la cadena productiva de la quinua, y entrelazarse bajo mecanismos de acción conjunta en la búsqueda de eficiencia colectiva (clúster). En ese contexto se busca la internacionalización de la quinua, por lo que impera que se diseñe una estrategia concreta, con políticas claras, definiendo objetivos en la agroexportación. Recordemos que la agroexportación está dejando como saldo un incremento porcentual considerable en el crecimiento económico de los países de Latinoamérica, trayendo como consecuencia incrementos en sus PBI. El crecimiento económico se está dando sobre la base de las exportaciones de productos no tradicionales en la última década, con mercados promisorios como Estados Unidos, Japón y países de Europa. En los últimos años se le está dando la adecuada importancia a este cultivo, dadas las excelentes cualidades alimenticias, nutritivas y tecnológicas (especialmente por su alto contenido en proteínas: 10,8\%-19,2\%, junto con la alta calidad de aminoácidos). La producción mundial de quinua está dada principalmente por Bolivia, Perú, Colombia y Ecuador. A inicios de la década de 1990 se estimaba en alrededor de 30 mil toneladas a escala mundial, y a fines de este periodo llegó a 50 mil toneladas. Más del 90\% de la quinua producida en el mundo proviene de Bolivia y Perú, exportando en mayor cantidad Bolivia (alrededor de 5 millones de dólares por año). Finalmente, en la región productora altoandina de Puno se busca lograr una mejor posición competitiva y un impacto socioeconómico que cubra las expectativas bajo los criterios planteados.

Los objetivos de la investigación están relacionados con la determinación del volumen de la oferta exportable y de los principales factores que inciden en la competitividad de la quinua perlada en Puno, 
así como las ventajas comparativa y competitiva de las operaciones de transformación y comercialización, cuyo desarrollo es de relevancia y prioridad para atender los nichos de mercado identificados en Estados Unidos, Canadá, Alemania, Australia y Japón.

\section{MATERIALES Y MÉTODOS}

\subsection{Zona de estudio}

La investigación se desarrolló en la región Puno, tomando la información de 1991 al 2012.

\subsection{Fuentes de información}

La información se obtuvo de fuentes primarias dentro de las zonas de producción y comercialización, en especial de las empresas involucradas en la cadena de valor y las instituciones gubernamentales y no gubernamentales relacionadas con la investigación, producción y comercialización de la quinua. La recopilación y obtención de información se realizó mediante entrevistas personales con los productores asociados o individualmente; con los comercializadores y con las personas o instituciones involucradas en la cadena de valor de la quinua. Por otro lado, se utilizó la técnica de la observación, tomándose apuntes de las acciones y hechos que fueron de interés a lo largo de la cadena de valor.

En lo que respecta a las fuentes secundarias, se obtuvo información de investigaciones similares recientes en la zona, descritas en tesis para la obtención de grado y posgrado, libros y revistas especializadas, publicaciones de conferencias y seminarios que se muestran en la bibliografía.

\subsection{Metodología}

La metodología utilizada para la investigación tomó como referencia los volúmenes de producción, los rendimientos, la tecnología y los aspectos socioeconómicos de la zona, así como los factores macroeconómicos para la exportación de la quinua, complementada con la información obtenida de las encuestas a los miembros del eslabón de la cadena, del trabajo de campo en plantas transformadoras, en las ferias y los talleres, para la interpretación, procesamiento, elaboración y manejo de la base de datos. 
- Fase 1. Recopilación de información.- Se recopiló y evaluó la información de las instituciones que desarrollan estadísticas de exportación de la quinua nacional e internacional, así como la información de las regiones agrarias de Puno y la información estadística del Ministerio de Agricultura (Minag).

- Fase 2. Aplicación de los modelos.- El estudio de competitividad se realizó con los modelos planteados de Porter: el análisis de la cadena valor, el análisis FODA y el análisis del diamante competitivo.

- El análisis de la cadena de valor: Mediante esta herramienta se determinó el valor de la quinua perlada como la suma de los beneficios percibidos menos los costos percibidos al vender la quinua. Con este método se identificó la fuente o las fuentes de la ventaja competitiva en las actividades que generan valor, descomponiendo sistemáticamente la agroindustria de la quinua perlada en cada una de las diferentes actividades, lo cual dio soporte para responder a la hipótesis general planteada. De existir estas fuentes de ventaja competitiva se aprecian las actividades existentes, que generan bajo costo y mejor diferenciación que sus rivales. Este modelo analizó también los márgenes que aportan ciertas actividades generadoras de valor. Se analizaron las actividades directas e indirectas así como las que reflejan el sistema de la calidad actual. En líneas generales, el uso de este método permite un análisis estratégico de costos y la determinación de la base para la diferenciación con otras regiones de Latinoamérica.

- El análisis FODA: Fue un pilar para el análisis interno y externo de la agroindustria de quinua. Mediante el análisis interno del FODA se examinaron las fortalezas, que ayudan a crear o desarrollar capacidades clave, y las debilidades, que obstaculizan la creación o el desarrollo de capacidades clave, y mediante el análisis externo se examinaron las oportunidades, las nuevas tendencias alimenticias (preferencia por lo orgánico, lo ecológico, lo estándar, la mejor fuente de proteína) que otros también lo quieren aprovechar; así como las amenazas, que consisten en las situaciones externas incontrolables pero previsibles. El contexto en que se desarrolla la quinua necesita de un análisis profundo de las fortalezas naturales o creadas por los agentes de la cadena, de las instituciones y del gobierno, que sirven para aprovechar las oportunidades y mantener una posición centrada ante las amenazas. 
- El análisis del diamante competitivo de Porter: Es un enfoque más completo y más interrelacionado con sus componentes al analizar una realidad. En este modelo, la ventaja competitiva de una región, Puno, fue el resultado de cuatro factores y actividades ligados entre las compañías que participan de los clústers, fundamentales para alcanzar la ventaja competitiva. Estos factores pueden estar influenciados de una manera proactiva por el gobierno. La estrategia, la estructura y la rivalidad entre las compañías es un factor que contribuye a crear reglas de juego claras y estables. En este aspecto, lo que se buscó fueron rivales regionales o se diseñó la estrategia para la formación de rivales regionales fuertes, que en el caso de Puno podría ser entre los agricultores de cada provincia o de cada zona o entre los productores del sur y del norte de Puno, para que haya una buena competencia dentro de la región. La condición de los factores describe elementos de ventaja comparativa que, en el caso de Puno, es bastante singular: mano de obra, tierra y capital, además de las condiciones agrológicas, climáticas y geográficas. En este aspecto lo que se buscó fue determinar si hay una correlación entre la dotación de recursos naturales y de desarrollo regional, y si existe especialización de los recursos humanos en determinadas tecnologías. Por otro lado, el factor determinante para este juego competitivo es encontrar la demanda por parte de consumidores condicionada por consumidores más exigentes cada día. El cuarto factor es tan importante como la condición de los factores y la exigencia de los consumidores. Al ser el clúster una agrupación de instituciones, empresas y organizaciones que contribuyen al desarrollo de un determinado sector, lo que se pretendió fue encontrar la función de los sectores conexos, ver si estos comparten información, resuelven problemas de procesamiento o promueven intercambios de ideas o innovaciones, al menos en quinua orgánica es una ventana para dar propulsión de nuevos paradigmas de producción agrícola.

\section{RESULTADOS Y DISCUSIÓN}

\subsection{Análisis de la cadena de valor}

Primero se analizó la producción agrícola de la quinua, la cual ha experimentado altibajos, tal como se aprecia en la tabla 1, y en los últimos 
22 años ha estado limitada a niveles máximos de siembra. La logística interna en la cadena es deficitaria, restándole competitividad. Por otra parte, las operaciones de campo son desarrolladas en un $80 \%$ con tecnologías de bajo nivel y en un $20 \%$ con un nivel de tecnología intermedio, cosechando también en el mismo orden.

Tabla 1

Evolución de la producción, la superficie y el rendimiento de la quinua en el Perú

\begin{tabular}{cccc}
\hline Campaña & $\begin{array}{c}\text { Superficie } \\
\text { (has) }\end{array}$ & $\begin{array}{c}\text { Producción } \\
\text { (toneladas) }\end{array}$ & $\begin{array}{c}\text { Rendimiento } \\
\text { (kg/ha) }\end{array}$ \\
\hline 1991 & 20.144 & 14.506 & 479 \\
1992 & 7.322 & 3.958 & 503 \\
1993 & 17.841 & 14.092 & 790 \\
1994 & 20.693 & 16.624 & 803 \\
1995 & 18.696 & 13.326 & 735 \\
1996 & 18.702 & 16.067 & 859 \\
1997 & 27.025 & 23.606 & 873 \\
1998 & 30.711 & 28.605 & 931 \\
1999 & 28.971 & 28.431 & 981 \\
2000 & 28.974 & 28.375 & 982 \\
2001 & 28.327 & 22.349 & 873 \\
2002 & 28.179 & 30.400 & 1.092 \\
2003 & 28.354 & 30.100 & 1.103 \\
2004 & 27.847 & 27.000 & 995 \\
2005 & $29.668,75$ & $32.610,5$ & 880 \\
2006 & $30.070,00$ & $29.002,3$ & 958 \\
2007 & $30.751,25$ & $30.196,5$ & 857 \\
2008 & $31.456,25$ & $26.548,5$ & 879 \\
2009 & $32.618,75$ & $39.572,2$ & 910 \\
2010 & $33.813,75$ & $51.282,67$ & 997 \\
2011 & $41.941,67$ & $41.083,55$ & 983 \\
2012 & $45.323,87$ & $44.167,22$ & 974 \\
\hline & & & \\
\hline
\end{tabular}

Fuente: Ministerio de Agricultura del Perú (1993-2012), DRA Puno, Sistema Integrado de Estadística Agraria (SIEA). 
Figura 1

Evolución de la producción de la quinua en el Perú

(toneladas)

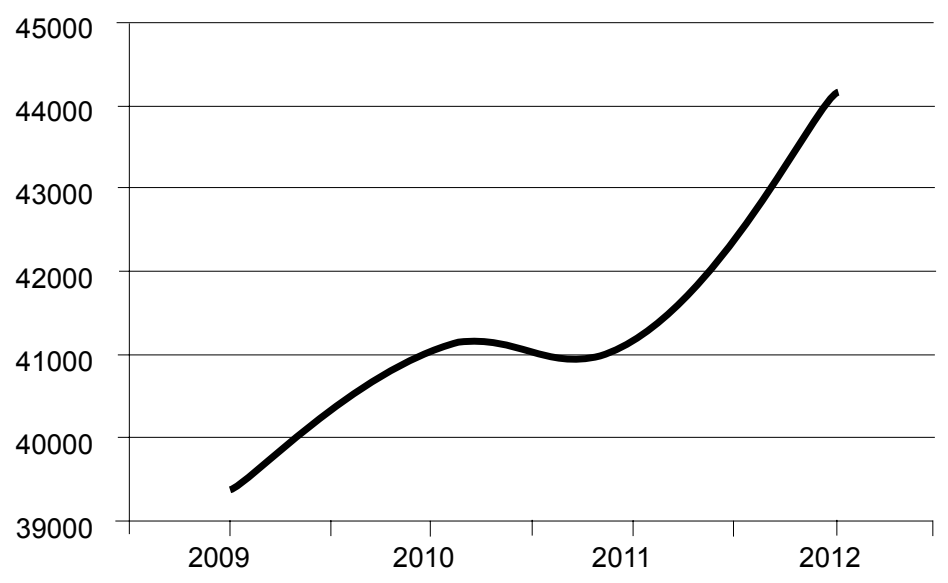

Fuente: Ministerio de Agricultura del Perú, 1991-2012.

Puno es el mayor productor de quinua. Se produce principalmente en las provincias de Puno (Acora, Capachica, Mañazo y Vilque), El Collao (Ilave); Chucuito (Juli, Pomata y Desaguadero), Yunguyo (norte de Puno), Azángaro (Arapa, Chupa, San José), Huancané (Taraco, Pusi), Lampa (Cabanilla, Pucara), Melgar (Ayaviri, Llalli, Nuñoa, Umachiri) y San Román (Cabana, Caracoto).

Tabla 2

Superficie cosechada, producción y rendimiento promedio de la quinua de las provincias del departamento de Puno (campaña agrícola 2012)

\begin{tabular}{llccc}
\hline \multicolumn{2}{l}{ Ámbito de agencias agrarias } & $\begin{array}{c}\text { Cosecha } \\
\text { (has) }\end{array}$ & $\begin{array}{c}\text { Rendimiento } \\
\text { promedio (kg/ha) }\end{array}$ & $\begin{array}{c}\text { Producción } \\
\text { (toneladas) }\end{array}$ \\
\hline El Collao & A.A. llave & $\mathbf{6 . 7 8 4}$ & 1.297 & 8.800 \\
Chucuito & Juli & $\mathbf{4 . 4 9 4}$ & 1.180 & 6.789 \\
Puno & SALC-Puno & 2.581 & 1.194 & 3.578 \\
San Román & Juliaca & 4.264 & 1.010 & 2.672 \\
Azángaro & Azángaro & $\mathbf{5 . 1 9 9}$ & 1.161 & 5.846 \\
\hline
\end{tabular}




\begin{tabular}{llccc} 
(continuación) & \multicolumn{3}{l}{} \\
\hline \multicolumn{2}{l}{ Ámbito de agencias agrarias } & $\begin{array}{c}\text { Cosecha } \\
\text { (has) }\end{array}$ & $\begin{array}{c}\text { Rendimiento } \\
\text { promedio (kg/ha) }\end{array}$ & $\begin{array}{c}\text { Producción } \\
\text { (toneladas) }\end{array}$ \\
\hline Lampa & Lampa & 903 & 997 & 1.557 \\
Melgar & Ayav-Llalli & 305 & 950 & 564 \\
Yunguyo & Yunguyo & 250 & 780 & 590 \\
Huancané & Taraco, Pusi & 1.556 & 1.030 & 1.727 \\
SA Putina & SA Putina & 585 & 500 & 740 \\
Moho & Moho & 115 & 600 & 119,1 \\
Carabaya & Carabaya & 15 & & 22 \\
Total & & $\mathbf{2 7 , 0 5 1}$ & & $\mathbf{3 2 . 0 0 4 , 1}$ \\
\hline
\end{tabular}

Fuente: Ministerio de Agricultura del Perú (2012).

\subsubsection{Producción y rendimientos de la quinua}

La producción de quinua pasó de 3.539 toneladas en 1990 a 14.095 toneladas en 1993 a escala nacional, donde Puno concentra el mayor volumen, con 10.616 toneladas. En el año 2012 se calcularon 44.167,22 toneladas en todo el país, y en la que se estima que Puno produjo 32.004 toneladas; le siguen Ayacucho $(5,8 \%)$ y Cusco $(4,7 \%)$ en capacidad productiva de este cereal. Estas regiones cuentan con condiciones agronómicas y climáticas adecuadas para el cultivo de la quinua, y son asistidas técnicamente por los gobiernos regionales y las organizaciones internacionales interesadas en su comercialización en el ámbito internacional.

En el 2009, la producción se incrementó debido al elevado número de hectáreas de cultivo y un mayor nivel de productividad por hectárea cosechada, resultado esto último de la asistencia técnica que vienen recibiendo determinados núcleos de producción. 


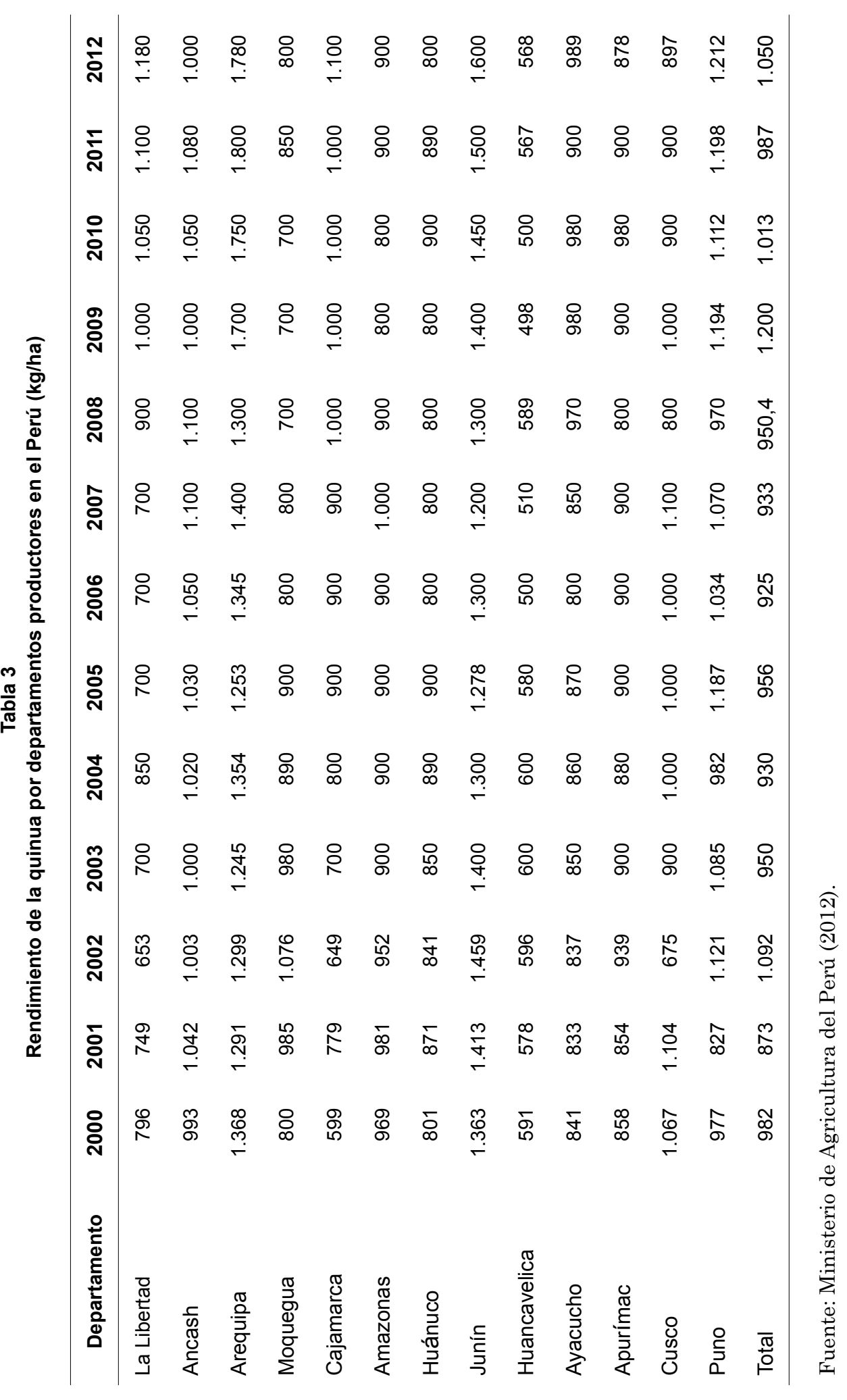


El distrito puneño de Cabana espera constituirse en el primer distrito productor de quinua de la región Puno. Al cierre del 2010 logró producir más de 600 toneladas, debido a que cuentan con una planta de procesamiento. El proyecto Desarrollo de Capacidades de la cadena productiva de quinua de Puno está dando frutos. El rendimiento promedio en el último año de análisis (2012) se situó en $1.212 \mathrm{~kg} / \mathrm{ha}$, el cual es mucho mayor que los rendimientos alcanzados anteriormente. En la región de Ayacucho la producción de quinua está bastante dispersa entre sus provincias, y además cuenta con extensiones pequeñas.

\subsubsection{Costos de producción}

En las tablas 4 y 5 se aprecian los costos de producción, correspondientes a diferentes niveles de tecnología dentro de zonas del país y entre los países productores a nivel mundial, según los factores productivos involucrados. Si bien estos costos reflejan el momento en que se tomó los datos, en la actualidad persisten los costos más bajos para la región Puno, y análogamente utilizando un nivel de tecnología baja el Perú sigue teniendo el costo más bajo dentro de los países productores de quinua. El $50 \%$ de sus costos es mano de obra.

Tabla 4

Costos de producción en los departamentos más representativos del Perú de acuerdo con una diferente tecnología (US\$/ha)

\begin{tabular}{lrrrrrrr}
\hline \multirow{2}{*}{ Componentes } & \multicolumn{2}{c}{ Puno } & \multicolumn{2}{c}{ Junín } & \multicolumn{2}{c}{ Cusco } & \multicolumn{2}{c}{ Promedio } \\
\cline { 2 - 8 } & \multicolumn{2}{c}{ Tecnología } & \multicolumn{2}{c}{ Tecnología } & Tecnología & \multicolumn{2}{c}{ Tecnología } \\
& Baja & Media & Media & Alta & Alta & Media & Alta \\
\hline $\begin{array}{l}\text { Preparación del } \\
\text { terreno }\end{array}$ & 17,14 & 64,29 & 60,00 & 30,00 & 71,00 & 62,14 & 50.715 \\
$\begin{array}{l}\text { Siembra y } \\
\text { fertilización }\end{array}$ & 70,00 & 11,43 & 100,00 & 85,70 & 28,57 & 55,71 & 57.135 \\
Labores culturales & 14,29 & 62,86 & 40,60 & 109,00 & 153,40 & 51,73 & 110.550 \\
Cosecha & 127,14 & 217,14 & 108,00 & 144,90 & 191,40 & 112,57 & 118.150 \\
Insumos & 8,54 & 38,57 & 222,80 & 261,60 & 287,10 & 75,69 & 204.350 \\
Otros & 26,00 & 184,29 & 143,20 & 221,00 & 265,80 & 113,74 & 193.400 \\
TOTAL & $\mathbf{2 6 3 , 1}$ & $\mathbf{5 7 8 , 5}$ & $\mathbf{6 7 4 , 6}$ & $\mathbf{8 8 0 , 2}$ & $\mathbf{9 1 2 , 7 0}$ & $\mathbf{6 2 6 , 5}$ & 896.45 .3 \\
\hline
\end{tabular}

Fuente: Ministerio de Agricultura, 2012. 
Tabla 5

Costos de producción de los principales países abastecedores (US\$/ha)

\begin{tabular}{|c|c|c|c|c|c|c|c|}
\hline \multirow{3}{*}{ Componentes } & \multicolumn{3}{|c|}{ Bolivia } & \multicolumn{3}{|c|}{ Perú } & \multirow{3}{*}{$\begin{array}{c}\text { Ecuador } \\
\text { Tecnología } \\
\text { Media }\end{array}$} \\
\hline & \multicolumn{3}{|c|}{ Tecnología } & \multicolumn{3}{|c|}{ Tecnología } & \\
\hline & Baja & Media & Alta & Baja & Media & Alta & \\
\hline $\begin{array}{l}\text { Preparación del } \\
\text { terreno }\end{array}$ & 95,00 & 78,50 & 148,50 & 17,14 & 62,14 & 50,71 & 208,00 \\
\hline Siembra y fertilización & 87,50 & 57,50 & 133,50 & 70,00 & 55,71 & 57,13 & 68,00 \\
\hline Labores culturales & 30,00 & 30,00 & 130,00 & 14,29 & 51,73 & 110,50 & 96,00 \\
\hline Cosecha & 45,00 & 99,00 & 139,00 & 127,14 & 112,57 & 118,10 & 205,00 \\
\hline Insumos & 40,25 & 50,25 & 210,25 & 8,57 & 75,69 & 204,30 & 12,90 \\
\hline Otros & 52,30 & 88,30 & 136,00 & 26,00 & 113,74 & 193,4 & 95,90 \\
\hline TOTAL & 350,1 & 413,5 & 700,2 & 263,1 & 626,55 & 896,45 & 685,80 \\
\hline
\end{tabular}

Fuente: Ministerios de Agricultura del Perú, de Bolivia y del Ecuador, 2002.

\subsubsection{Comercialización y exportación}

Una de las partes más importantes de la cadena principal del negocio de la quinua es sin duda el acopio y la comercialización de la quinua. En el altiplano peruano, los comerciantes mayoristas de Juliaca son los principales agentes de abastecimiento a otros mercados y al procesamiento local. En estos últimos años los márgenes de utilidad han ido mejorando para el productor y existe un buen margen para el exportador (véase la tabla 7).

La cadena productiva de la quinua es aún incipiente y compleja por la alta intermediación, lo cual ocasiona una dispersión de precios. El primer eslabón de comercialización lo conforman las comunidades andinas, integradas por pequeños agricultores sin poder de negociación, y algunos agricultores con más de $3 \mathrm{ha}$, considerados grandes para los fines de este artículo. Siguen los acopiadores, que cuentan con gran poder de negociación por manejar el efectivo, haber hecho contratos previos o haber financiado la campaña.

Los mayoristas se encuentran por lo general en la ciudad de Juliaca, de la región Puno; al igual que los acopiadores, mantienen un alto poder de negociación. Las empresas procesadoras se encuentran en Juliaca, sin embargo en los últimos años estas también se han incrementado en las ciudades de Arequipa y Lima, pues tratan de concentrar la mayor cantidad de acopiadores y mejorar el estándar del grano, al 
mezclar variedades y optimizar el proceso del perlado (limpieza, selección, lavado, secado y empacado) para exportar una quinua de calidad homogénea, al menos en el tamaño.

Tabla 6

Exportaciones de quinua (partida arancelaria 10089019.00)

\begin{tabular}{lccccccccc}
\hline Años & $\mathbf{2 0 0 3}$ & $\mathbf{2 0 0 4}$ & $\mathbf{2 0 0 5}$ & $\mathbf{2 0 0 6}$ & $\mathbf{2 0 0 7}$ & $\mathbf{2 0 0 8}$ & $\mathbf{2 0 0 9}$ & $\mathbf{2 0 1 0}$ & $\mathbf{2 0 1 1}$ \\
\hline $\begin{array}{l}\text { Millones de } \\
\text { dólares }\end{array}$ & 0,4 & 0,4 & 0,7 & 1,5 & 2,0 & 5,1 & 7,1 & 13,1 & 25 \\
$\begin{array}{l}\text { Miles de } \\
\text { toneladas }\end{array}$ & 0,3 & 0,3 & 0,6 & 1,3 & 1,5 & 2,1 & 2,7 & 4,8 & 7,9 \\
\hline
\end{tabular}

Fuente: Sunat (2012).

La exportación ha crecido sostenidamente a partir del 2005 hasta el 2011, en este periodo se ha incrementado con una tendencia dinámica de $86 \%$. Para el año 2011 ha crecido en $92 \%$, hasta US $\$ 25$ millones, en comparación con los US $\$ 13$ millones reportados en el mismo periodo del año anterior; este incremento se debe a la mayor demanda existente, por su extraordinaria calidad nutricional y gastronómica.

En el 2011, Estados Unidos se convirtió en el principal destino de la quinua peruana al demandar más de 15 millones, concentrando el 61\% del total de envíos. La quinua goza de acceso preferencial libre de pago de aranceles a sus principales mercados de destino: Estados Unidos, Alemania, Italia, Canadá y Australia.

En el comercio mundial las exportaciones peruanas junto a las bolivianas han representado durante el 2011 un total de 24 mil toneladas, por un valor FOB de 72,3 millones de dólares, de esta cifra el $28 \%$ es peruano.

La empresa Exportadora Agrícola Orgánica y la empresa Grupo Orgánico Nacional concentraron el 53,8\% del valor de las exportaciones en el 2010. Las empresas que integran la oferta exportable de quinua se encuentran en la búsqueda de nuevos mercados, intensificando la difusión de los beneficios del cereal, además de presentar variedades que vayan de acuerdo con las exigencias del mercado internacional, como variedades nativas y gourmet dirigidas al segmento de restaurantes y servicios de catering (quinua roja). 


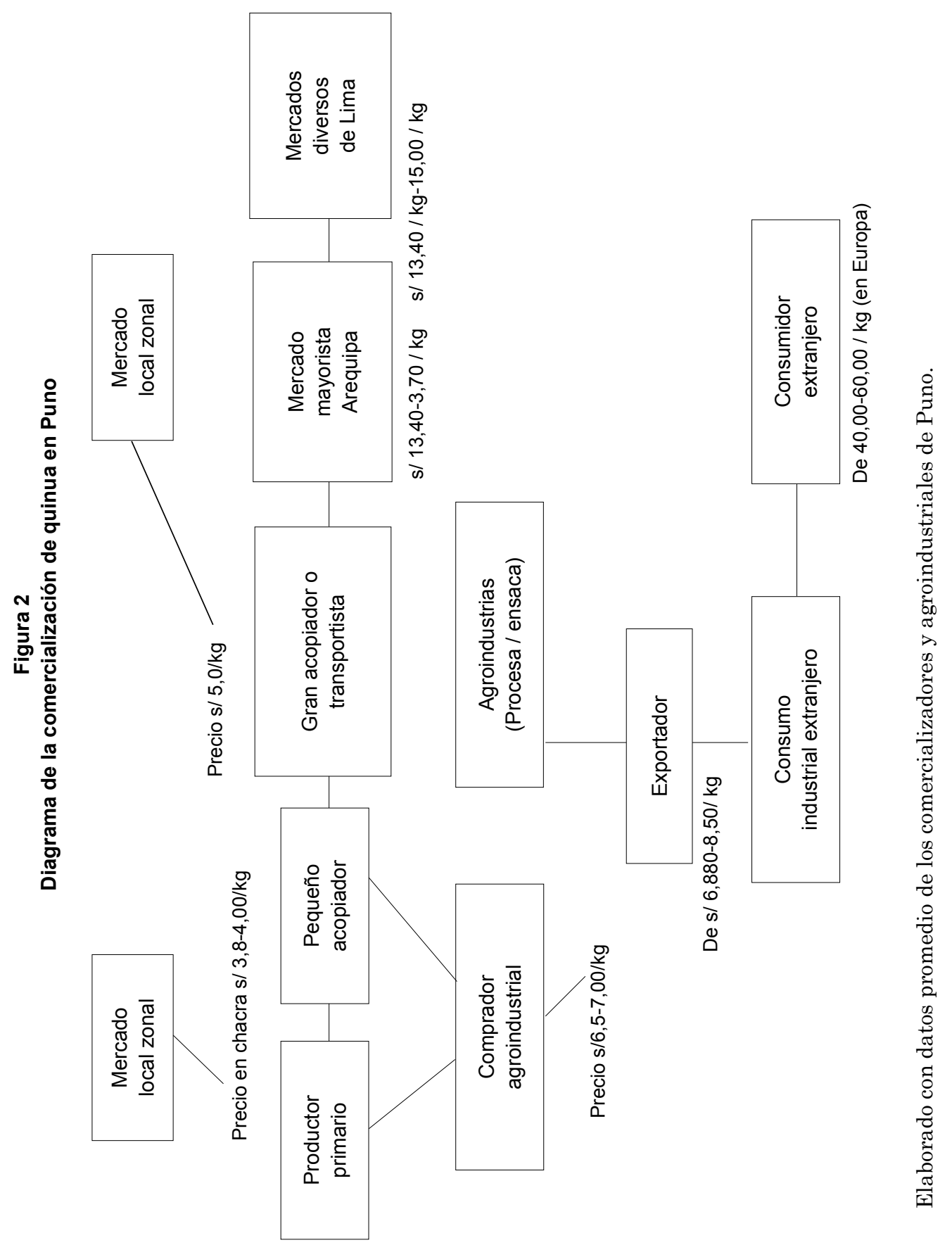

Ingeniería Industrial n. ${ }^{\circ}$ 31, 2013 
Tabla 7

Márgenes de comercialización, bruto y neto, de la quinua perlada en el altiplano de Puno (2012)

\begin{tabular}{lcccr}
\hline & $\begin{array}{r}\text { Precios } \\
\mathbf{( s / . / k g )}\end{array}$ & $\begin{array}{r}\text { Costos } \\
\mathbf{( s / . / k g )}\end{array}$ & $\begin{array}{c}\text { Margen } \\
\text { bruto (\%) }\end{array}$ & $\begin{array}{r}\text { Margen } \\
\text { neto (\%) }\end{array}$ \\
\hline Productor peq./mediano & 3,80 & 1,64 & 36,80 & 22,80 \\
Productor grande & 4,04 & 1,60 & 36,80 & 24,40 \\
Pequeño acopiador & 5.00 & 0,90 & 2,40 & 6,00 \\
Gran acopiador & 4,80 & 0,50 & 16,60 & 6,25 \\
Agroindustria & 6,50 & 1,00 & 26,15 & 10,76 \\
Exportador & 8,50 & 3,90 & 23,52 & 9,40 \\
\hline Total intermediación & & & 55,30 & 32,41 \\
\hline
\end{tabular}

Fuente: Comercializadores de Puno (2012). Datos tomados de los agroindustriales de la ciudad de Juliaca.

\subsection{Análisis del desarrollo del diamante competitivo de Porter}

La competitividad del Perú en el sector agroindustrial, específicamente de la quinua perlada para la exportación, depende del contexto que rodea a las empresas que conforman este sector. En la figura 3 se detalla el análisis del diamante competitivo de Porter en la cadena agroindustrial de quinua perlada para exportación. Este análisis permitió resumir claramente los factores básicos: recursos naturales, recursos humanos, recursos de capital, infraestructura de la información, infraestructura institucional, y como factores avanzados: la infraestructura científica y técnica y la infraestructura física, que inciden en este sector. Así también, se describieron las condiciones de la demanda y la estrategia, estructura y rivalidad de las empresas, influidas por los clústers o sectores conexos surgidos en torno al rubro de exportación. Se incluyeron en forma desagregada aspectos relacionados de manera interdependiente en cada parte del diamante, algunos de ellos con alta persistencia y otros con un nivel de superación iniciada. Como ejemplo podemos mencionar que el gobierno está allanando el camino para el logro de los objetivos estratégicos, y, por otro lado, las condiciones de los diversos factores: productivos, organizativos, de gestión, de investigación, de mejoras tecnológicas, de calidad, que se van a ir dando y mejorando en la medida en que las metas tácticas y operativas se vayan logrando. 
Tabla 8

Análisis FODA de la producción y comercialización de la quinua

\begin{tabular}{ll}
\hline \multicolumn{1}{c}{ Fortalezas } & \multicolumn{1}{c}{ Debilidades } \\
\hline $\begin{array}{l}\text { Productividad promedio mayor a la regis- } \\
\text { trada en Bolivia }\end{array}$ & $\begin{array}{l}\text { Escasa asistencia técnica. La mayor par- } \\
\text { te de la quinua convencional es procesa- } \\
\text { da en forma artesanal }\end{array}$ \\
\hline $\begin{array}{l}\text { Condiciones agrícolas y de cultivo para } \\
\text { la producción de quinua a nivel regional }\end{array}$ & $\begin{array}{l}\text { Limitado acceso al financiamiento de los } \\
\text { agricultores }\end{array}$ \\
\hline $\begin{array}{l}\text { Cereal con alto valor nutritivo, su valor } \\
\text { calórico es superior al de otros cereales } \\
\text { (350 cal/100 g) }\end{array}$ & Alta intermediación del mercado local \\
\hline $\begin{array}{l}\text { Desarrollo de nuevas variedades y téc- } \\
\text { nicas mejoradas para elevar la produc- } \\
\text { tividad }\end{array}$ & $\begin{array}{l}\text { Poca difusión de los beneficios nutricio- } \\
\text { nales en el mercado local }\end{array}$ \\
\hline $\begin{array}{l}\text { Condiciones orgánicas de producción } \\
\text { Bresencia de cooperación nacional e in- } \\
\text { ternacional para el desarrollo productivo } \\
\text { y comercial de la quinua }\end{array}$ & $\begin{array}{l}\text { La quinua considerada como cultivo de } \\
\text { autoconsumo en las regiones de la sierra }\end{array}$ \\
\hline
\end{tabular}

Elevada concentración de las exportaciones hacia Estados Unidos

Escaso nivel de organización de los productores ante las exigencias del mercado internacional de calidad estandarizada

Matriz de oportunidades y amenazas

\begin{tabular}{ll}
\hline \multicolumn{1}{c}{ Corto plazo } \\
\hline \multicolumn{1}{c}{ Oportunidades } & \multicolumn{1}{c}{ Amenazas } \\
\hline $\begin{array}{l}\text { Creciente demanda de materia prima } \\
\text { orgánica e insumos para la industria de } \\
\text { transformación de alimentos }\end{array}$ & $\begin{array}{l}\text { Bajo desarrollo de productos derivados de } \\
\text { la quinua con fines de exportación }\end{array}$ \\
\hline $\begin{array}{l}\text { Precios pagados en mercados euro- } \\
\text { peos y estadounidense por tonelada de } \\
\text { quinua orgánica y real son elevados. }\end{array}$ & $\begin{array}{l}\text { Apreciación de la moneda local frente al } \\
\text { dólar }\end{array}$ \\
\hline
\end{tabular}


(continuación)

\begin{tabular}{ll}
\hline \multicolumn{1}{c}{ Corto plazo } \\
\hline \multicolumn{1}{c}{ Oportunidades } & \multicolumn{1}{c}{ Amenazas } \\
\hline $\begin{array}{l}\text { Bolivia, el mayor exportador mundial, ha } \\
\text { perdido sus preferencias arancelarias } \\
\text { con Estados Unidos (Atpdea) }\end{array}$ & $\begin{array}{l}\text { Mayores envíos de quinua boliviana a Es- } \\
\text { tados Unidos y Europa }\end{array}$ \\
\hline $\begin{array}{l}\text { Dentro de la categoría de acompaña- } \\
\text { mientos y almidones, la quinua se ubicó } \\
\text { en el segundo lugar como la tendencia } \\
\text { en boga en el 2011 en los restaurantes } \\
\text { estadounidenses }\end{array}$ & $\begin{array}{l}\text { Mayores compras de quinua peruana por } \\
\text { parte de }\end{array}$ \\
\hline $\begin{array}{l}\text { Crecimiento de la la demara res de la región } \\
\text { cereales andinos envasados }\end{array}$ & \\
\hline
\end{tabular}

Las oportunidades y las amenazas son de alto impacto y alta probabilidad.

\begin{tabular}{ccc}
\hline & Mediano plazo & \\
\hline Oportunidades & Amenazas \\
\hline
\end{tabular}

Tendencia creciente del mercado orgá- Costos elevados en el desarrollo de una nico en Europa y Centroamérica. marca propia del mercado europeo

Ingreso al mercado coreano tras la firma del TLC con ese país

Ingreso al mercado brasileño, luego del análisis del riesgo de plagas

Bolivia, único proveedor de quinua de Brasil, introduciría nuevas estrategias para obtener mayor participación en el mercado

Promoción de la organización, la producción y el consumo de la quinua a través de gobiernos regionales

La Organización Mundial de la Salud (OMS) ha considerado a la quinua como uno de los alimentos recomendados para el futuro.

Un mercado potencial para la quinua es el de los celiacos $(0,5 \%$ de la población mundial), ya que este cereal no contiene gluten

Las oportunidades y amenazas a mediano plazo son de alto impacto; siendo de alta probabilidad las tres primeras y de media probabilidad las tres últimas 
Figura 3

Diamante competitivo del sector quinua (Puno) 2012

\section{Gobierno}

- Escasa promoción de las exportaciones

- Creación de ceticos

- Centros de investigación y capacitación

- Investigación de quinua para

exportación es deficiente

- Infraestructura de transporte

- Limitada accesibilidad de crédito

Financiamiento

- Marco regulatorio

- Prorroga de exoneración del IGV

- Libre disponibilidad de tierra

\section{Estrategia, estructura y rivalidad}

- Empresas de producción y exportación no asociadas ni articuladas entre sí

- Liderazgo de las organizaciones poco desarrolladas. Organizaciones y asociaciones débiles administrativamente

- Precios altos para la quinua orgánica

- La mayor parte de la producción es para el autoconsumo (60\%)

- No hay una estrategia de oferta organizada de largo plazo

- No existe marca registrada

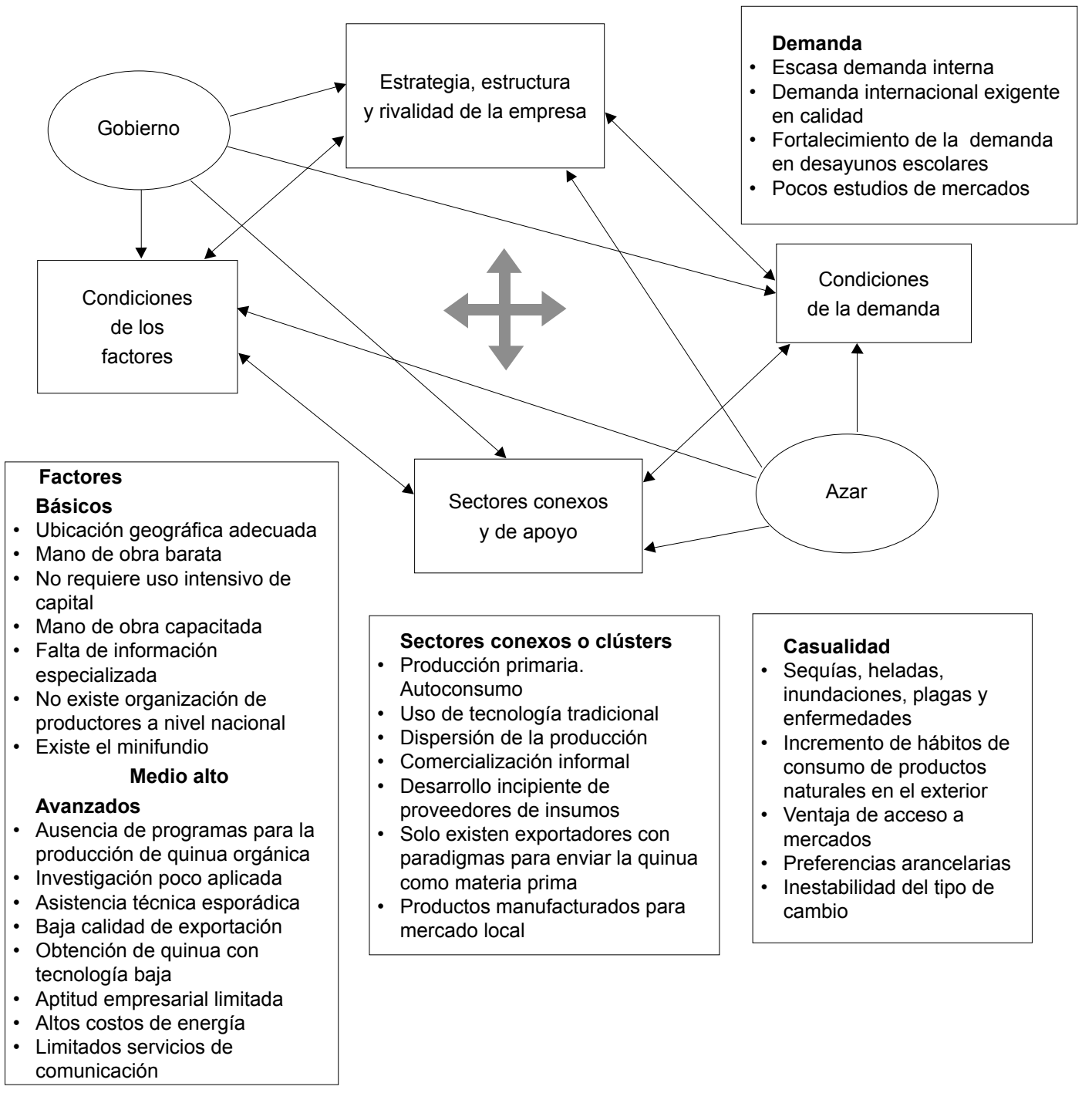

Elaboración propia. 
Figura 4

Flujo de la cadena exportadora futura de la quinua en Puno

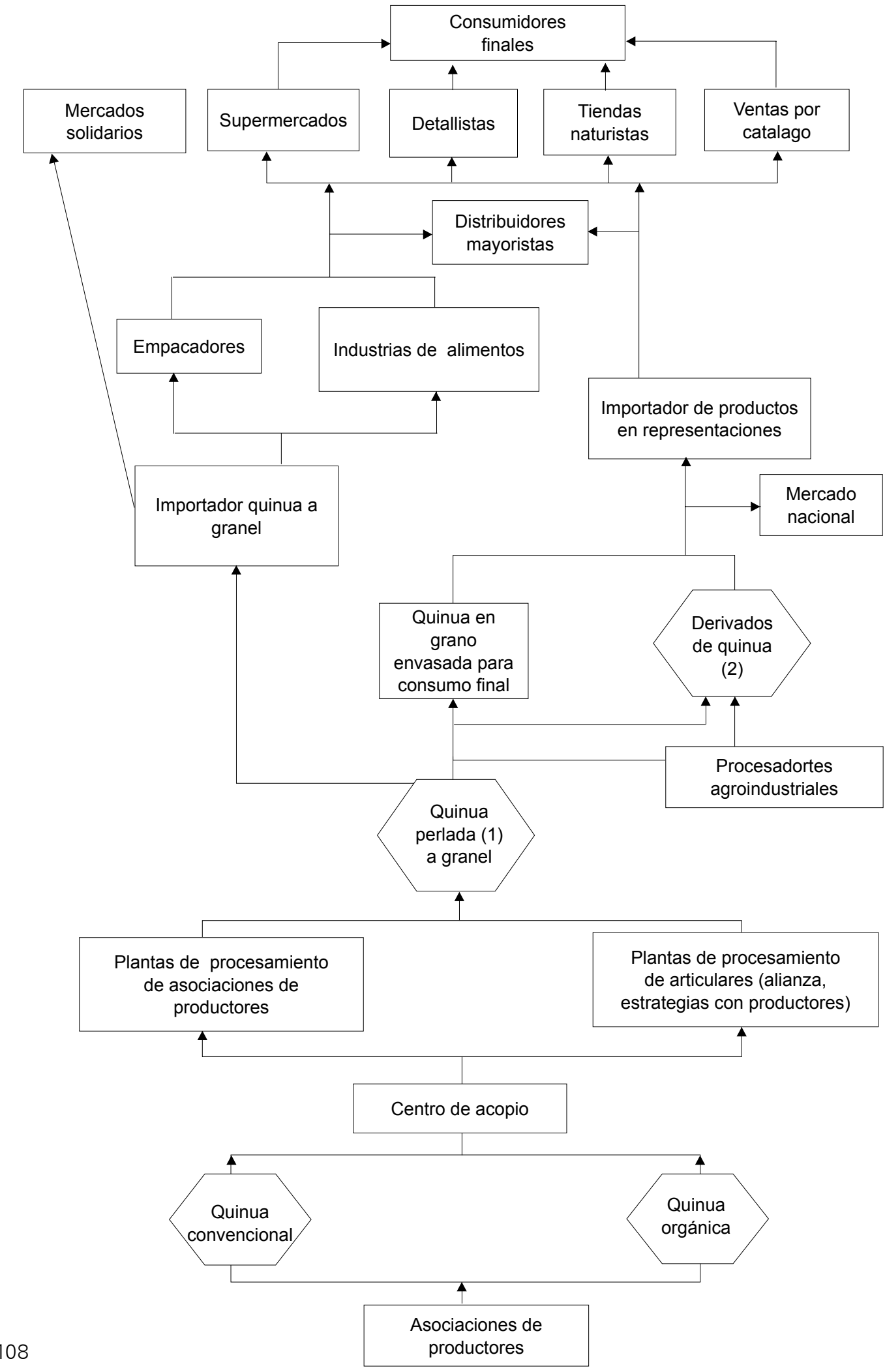




\subsection{Estrategias}

A continuación se presenta una lista de las principales estrategias para mejorar la competitividad en la producción y comercialización de la quinua:

1. Formar alianzas entre el productor y el exportador, para dar a los productores información sobre demanda y expectativa de la quinua en los mercados internacionales.

2. Financiar al sector de la quinua por medio de la banca privada en el mediano y el largo plazo, pero de manera indirecta, con aporte en semillas, capacitaciones, con el alquiler de maquinarias, identificando las necesidades de implementación de la asistencia técnica básica y avanzada.

3. Fomentar la transferencia de tecnología a través del Instituto Nacional de Innovación Agraria (INIA-Puno) como institución nacional de difusión.

4. Establecer equipos técnicos de trabajo que identifiquen y prioricen las necesidades de riego, forestación y fertilización orgánica.

5. Estrechar lazos con países consumidores y transformadores de la quinua para transferir (importar) tecnología, mediante convenios bilaterales.

6. Promover y reconocer las funciones que cumple la quinua en las comunidades andinas (función de seguridad alimentaria y función económica), respetando los patrones culturales y las formas de organización y de producción de dichas comunidades.

\section{CONCLUSIONES}

1. En el año 2012, la producción de quinua en Puno fue de 32.004,1 toneladas, de las cuales el $50 \%$ fue para autoconsumo, el $10 \%$ se derivó a la agroindustria local y regional y el $40 \%$ restante se dirigió al consumo directo, del cual, entre el $20 \%$ y el $25 \%$, se derivó a la exportación.

2. La demanda de quinua en el mercado internacional es de alrededor de 8.000 a 10.000 toneladas, lo cual es interesante para la florecien- 
te agroindustria nacional, pues hay por cubrir una brecha de entre 7.500 a 9.500 toneladas de quinua, la que actualmente es asistida por Bolivia y Ecuador.

3. Existe una ventaja competitiva en cuanto a los rendimientos. En el promedio nacional se obtiene entre $900 \mathrm{~kg} / \mathrm{ha}$ a $1.200 \mathrm{~kg} / \mathrm{ha}$ en los años analizados, y en Puno en el 2012 el rendimiento fue de alrededor de $1.212 \mathrm{~kg} / \mathrm{ha}$, superior al del principal exportador, Bolivia, donde su rendimiento es de $700 \mathrm{~kg} / \mathrm{ha}$.

4. Los principales factores que restringen una mayor oferta son las condiciones climáticas extremadamente duras, que limitan las opciones agropecuarias, y el uso limitado de la tierra, ya sea por falta de rotación de cultivo, de fertilización o de organización al momento de producir.

5. Las actividades de acopio y comercialización no tienen un alto nivel competitivo, debido a la deficiente organización por parte de los agentes económicos en esta parte de la cadena. Las actividades de transformación presentan ventajas competitivas, al menos hasta la obtención de granos perlados (secos, uniformes, limpios y sin saponina).

6. Las ventajas y beneficios son mayores que las desventajas en los acuerdos de libre comercio para la quinua, al estar dentro de contextos preferenciales arancelarios o de acuerdos bilaterales, como el Atpdea, que trae beneficios a los exportadores de la quinua hacia Estados Unidos, así como los TLC con países orientales.

\section{REFERENCIAS}

Adex (2012). Informe estadístico. Lima: Adex.

Agrodata (2012). Mercado de alimentos en Bolivia. La Paz: Usaid/ Cosude.

Alarcón, J. (2002). Mercadeo de productos agropecuarios. Teoría y aplicación al caso peruano. Lima: UNALM.

Bueno, E. (1995). Dirección estratégica de la empresa. Metodología técnica y casos. Madrid: Pirámide.

Camacho, A., \& Torres, H. (1980). Planta procesadora de quinua. Proyecto realizado para el Programa Andino de Desarrollo 
Tecnológico en el Área de Alimentos, en conjunto con el IICA. Puno.

Cillóniz, A. (1994). I Encuentro Nacional por la Agricultura Productiva: Fundamento Paz y Desarrollo. Lima: IPAE.

Egoávil, M., \& Reinoso, J. (1976). Los canales, costos y márgenes de comercialización de la quinua. Proyecto de Fondo Simón Bolivar a través del Ministerio de Agricultura y Alimentación de Perú. Lima: Instituto Interamericano de Ciencias Agrícolas.

ESAN (1998). Creación de valor y gerencia. Convención Empresarial. Lima: ESAN.

Gómez, J. (1997). Estrategias para la competitividad de las PYME. México D.F.: McGraw-Hill.

Gonçalves, J. (1999). Antecedentes para evaluar la competitividad de productos frutihortícolas de los países del Cono Sur. Grupo ONUFAO. São Paulo.

Gorbitz, A., \& Luna, R. (1953). Estudios sobre la quinua en el Perú. Lima: Ministerio de Agricultura, Programa Cooperativo de Experimentación Agropecuaria.

Hernández, R., Fernández, C., \& Baptista, P. (1998). Metodología para la investigación. México D. F.: McGraw-Hill.

Hill, Ch. (1995). Administración estratégica. Bogotá: McGraw-Hill.

IICA (1990). Comercialización de productos agropecuarios. Lima.

Mercado, E. (1997). Productividad, base de la competitividad. México D. F.: Limusa.

Meyhuay, M. (1999). Quinua. Composición y valor nutricional, usos, producción, importación, exportación, cosecha y poscosecha. Lima: Instituto de Desarrollo Agroindustrial.

Minag. Compendios estadísticos 1991-2000; 2001-2012; 2013-febrero. Lima.

Monitor Company (1995). Construyendo las ventajas competitivas del Perú. Los agronegocios. Lima: Promperú/Imagen y Medios.

Mujica, A., Seven, J., Izquierdo, J., \& Piere, J. (2001). Prueba americana y europea de quinua. Resultados (Chenopodium quinua willd). Documento FAO. Roma. 
Pari, D. (2003). Análisis de las ventajas competitivas de quinua peruana para la exportación - Puno. (Tesis para la obtención de Magister Scientiae en Economía Agrícola). Lima: Universidad Nacional Agraria La Molina.

Parkin, M. (2001). Microeconomía. México D. F.: Marisa de Anta.

Porter, M. (1987). Ventaja competitiva. México D. F.: Continental.

Porter, M. (1990). El comercio internacional. México D. F.: Continental.

Prompex (1998). ¿Cómo exportar? Curso de actualización para exportadores. Lima: Prompex.

Tapia, M. (1979). La quinua y la kañiwa. Bogotá: IICA.

Vásquez, V., \& Alza, M. (1996). Agroexportación. Análisis y perspectivas de productos no tradicionales. Rentabilidad, mercado y zonas de producción. Proyecto de Producción de Medios de Comunicación y Transferencia. Lima.

Villamizar, R., \& Mondragón, J. (1995). Lecciones de los países del AsiaPacífico en tecnología, productividad y competitividad. Bogotá: Norma.

Zurita Puma, D. (1992). Evaluación de la gestión comunal de empresas comunales en Puno: Zona Buenaventura. (Tesis para la obtención del Magister Scientiae en Economía Agrícola). Lima: Universidad Nacional Agraria La Molina. 